\title{
Editorial
}

\section{Theoretical and Computational Advances in Nonlinear Dynamical Systems}

\author{
Zhi-Yuan Sun, ${ }^{1}$ Panayotis G. Kevrekidis, ${ }^{2}$ Xin Yu, $^{3}$ and K. Nakkeeran ${ }^{4}$ \\ ${ }^{1}$ Department of Physics, Technion-Israel Institute of Technology, 320000 Haifa, Israel \\ ${ }^{2}$ Department of Mathematics and Statistics, University of Massachusetts, Amherst, MA 01003, USA \\ ${ }^{3}$ School of Aeronautic Science and Engineering, Beihang University, Beijing 100191, China \\ ${ }^{4}$ School of Engineering, University of Aberdeen, Aberdeen AB24 3UE, UK
}

Correspondence should be addressed to Zhi-Yuan Sun; sunzhiyuan137@aliyun.com

Received 4 May 2017; Accepted 4 May 2017; Published 4 June 2017

Copyright (C) 2017 Zhi-Yuan Sun et al. This is an open access article distributed under the Creative Commons Attribution License, which permits unrestricted use, distribution, and reproduction in any medium, provided the original work is properly cited.

\section{Introduction}

The theory of dynamical systems is a paradigm for studying various scientific phenomena, ranging from complex atomic lattices to planetary motion, from water waves to weather systems, from chemical reaction to biological behaviors, and many more. Relevant applications have widely arisen in multidisciplinary fields including mathematics, physics, chemistry, biology, and even economics and sociology. Due to the rapid development of theoretical and computational techniques in recent years, the role of nonlinearity in dynamical systems has attracted increasing interest and has been intensely investigated. Typical research areas include spatial and temporal evolution of nonlinear systems, pattern formation and their interactions, localized solutions and stability analysis, and many others. At the same time, the mathematical tools, for both of the symbolic and numerical aspects, have been developed in dealing with the nonlinear dynamical systems qualitatively and quantitatively. On the other hand, complexity of the nonlinear dynamical systems can be further portrayed when chaotic and stochastic behaviors are revealed. Interplay between nonlinearity and randomness is also a highlight topic which can be simulated and studied by modern computational resources.

Focusing on the nonlinearity of dynamical systems, this special issue has received more than 30 submissions, 12 papers of which have been accepted after strict review process. These papers cover works from theoretical analysis to applications in chaotic systems, fluid dynamics, solid mechanics, and stochastic and economical systems. Mathematical methods to analyze and obtain exact solutions for types of nonlinear dynamical systems have been included in our issue as well. A brief summary will be presented in the following sections. We expect these articles, including their substantial bibliographic resources, to be of great interest to the scholars in the relevant scientific and engineering communities.

\section{Chaotic Systems and Networks}

In their article "A Chaotic System with an Infinite Number of Equilibrium Points: Dynamics, Horseshoe, and Synchronization," the coauthors V.-T. Pham et al. introduce a new chaotic system which has hidden chaotic attractors with an infinite number of equilibrium points. They study dynamical properties of such a system via equilibrium analysis, bifurcation diagrams, and maximal Lyapunov exponents. The findings of topological horseshoes for this system are presented as well. The authors also investigate the possibility of synchronizing two new chaotic systems with infinite equilibria by using adaptive control.

In their article "Adaptive Modified Function Projective Lag Synchronization of Memristor-Based Five-Order Chaotic Circuit Systems," the coauthors Q. Li and S. Liu investigate the modified function projective lag synchronization of the memristor-based five-order chaotic circuit system with unknown bounded disturbances. They use the linear matrix inequality approach and Lyapunov stability theorem to establish an adaptive control law, which makes 
two different chaotic states asymptotically synchronized up to a desired scaling function matrix. The simulation is performed to show the correctness and effectiveness of their control method.

In his/her article "Asymptotic Stability and Asymptotic Synchronization of Memristive Regulatory-Type Networks," the author J.-E Zhang investigates nonlinear dynamics of memristive networks. The author studies global asymptotic stability and global asymptotic synchronization for memristive regulatory-type networks, based on the $M$-matrix theory and Lyapunov stability theory. Simulations are also performed to support the theoretical results.

\section{Fluid and Atmosphere Dynamics}

In their article "Numerical Simulations of the Square Lid Driven Cavity Flow of Bingham Fluids Using Nonconforming Finite Elements Coupled with a Direct Solver," the coauthors R. Mahmood et al. perform numerical simulations in a single and double lid driven square cavity to study the flow of a Bingham viscoplastic fluid. Their implementations are done via finite element methods in the framework of a monolithic approach, and the results are obtained for Bingham numbers in the range of $0-500$. They find that the number of iterations for Newton's method increases at large values of Bingham number due to enhanced nonlinearity.

In their article "Application of Adjoint Data Assimilation Method to Atmospheric Aerosol Transport Problems," the coauthors Minjie Xu et al. employ the adjoint assimilation method with the characteristic finite difference scheme to solve the atmospheric aerosol transport problem. Effectiveness of the method is shown in computing a Gaussian hump, and a better agreement with the ideal initial distribution is derived by using their adjoint method. In addition, a real case of $\mathrm{PM}_{2.5}$ concentration distribution in China during the APEC 2014 is simulated and analyzed in this work.

\section{Solid Mechanics}

In their article "The Spreading Residue Harmonic Balance Method for Strongly Nonlinear Vibrations of a Restrained Cantilever Beam," the coauthors Y. H. Qian et al. study a fifth-order nonlinear problem that describes the strongly nonlinear vibration of an elastically restrained beam with a lumped mass. They employ the spreading residue harmonic balance method to derive approximate analytical solutions for such a system. Illustrative examples are provided to verify the accuracy of their method, and their solutions are compared with the ones obtained by using other typical methods, as well as some exact solutions.

In their article "Closed-Form Exact Solutions for the Unforced Quintic Nonlinear Oscillator," the coauthors A. Beléndez et al. obtain closed-form exact solutions for the periodic motion of a one-dimensional, undamped, quintic oscillator. They consider all possible combinations of positive and negative coefficients of the linear and quintic terms, which provides four different cases but only three different pairs of periodic solutions. Some particular cases are discussed with variation of the parameters, and the period is presented to be a function of the initial amplitude. The authors also consider oscillatory motions around the nonzero equilibrium point.

\section{Stochastic System}

In their article "Turing Bifurcation and Pattern Formation of Stochastic Reaction-Diffusion System," the coauthors Q. Zheng et al. study spontaneous pattern formation induced by noise. They present a method to solve a stochastic reactiondiffusion system and show how the Turing bifurcation and Hopf bifurcation arise through linear stability analysis of the local equilibrium. They also develop the amplitude equation with a pair of wave vectors by using the Taylor series expansion, multiscaling, and expansion in powers of small parameters. In addition, the authors point out that their analytical results agree with numerical simulations.

\section{Mathematical Aspects}

In their article "The General Solution of Differential Equations with Caputo-Hadamard Fractional Derivatives and Noninstantaneous Impulses," the coauthors X. Zhang et al. study a Caputo-Hadamard fractional differential equation with noninstantaneous impulses. They obtain an equivalent integral equation with some undetermined constants, which indicates the existence of general solution for this impulsive system. An example is also given to illustrate their results.

In their article "The Convergence Ball and Error Analysis of the Relaxed Secant Method," the coauthors R. Lin et al. propose a relaxed secant method. A radius estimate of the convergence ball of such a method is obtained for the nonlinear systems with Lipschitz continuous divided differences of the first order. The error estimate is also established with the matched convergence order. The authors discuss the relation between the radius and the speed of convergence with parameters and present some numerical examples.

In their article "Error Estimates on Hybridizable Discontinuous Galerkin Methods for Parabolic Equations with Nonlinear Coefficients," the coauthors M. Moon et al. perform error estimations of the hybridizable discontinuous Galerkin method for parabolic equations with nonlinear coefficients. They give the bounds for their estimates when the nonlinear coefficients obey the Lipschitz condition. The authors also prove that the errors for their estimations are bounded.

\section{Economical System}

In their article "Computation of the Stability and Complexity about Triopoly Price Game Model with Delay Decision," the coauthors Y. Wang et al. develop a price game model based on the entropy theory and chaos theory. They consider the three enterprises bounded rationality and use the cost function under the resource constraints. With variation of the delay parameters, bifurcation, stability, and chaos of the system are discussed, and the change of entropy is considered when the system is far away from its equilibrium. They also show that chaos of the system can be controlled effectively. 


\section{Acknowledgments}

We would like to express our sincere thanks to all the authors and reviewers for their contributions to this special issue.

Zhi-Yuan Sun

Panayotis G. Kevrekidis

Xin $\mathrm{Yu}$

K. Nakkeeran 


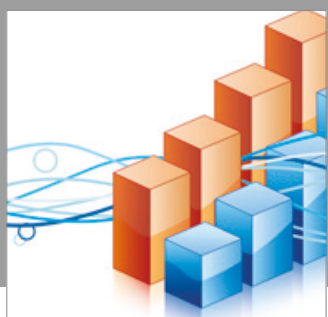

Advances in

Operations Research

vatersals

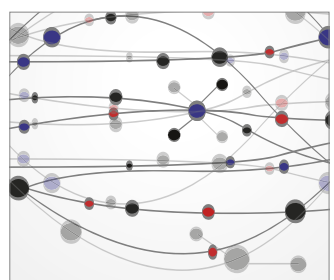

\section{The Scientific} World Journal
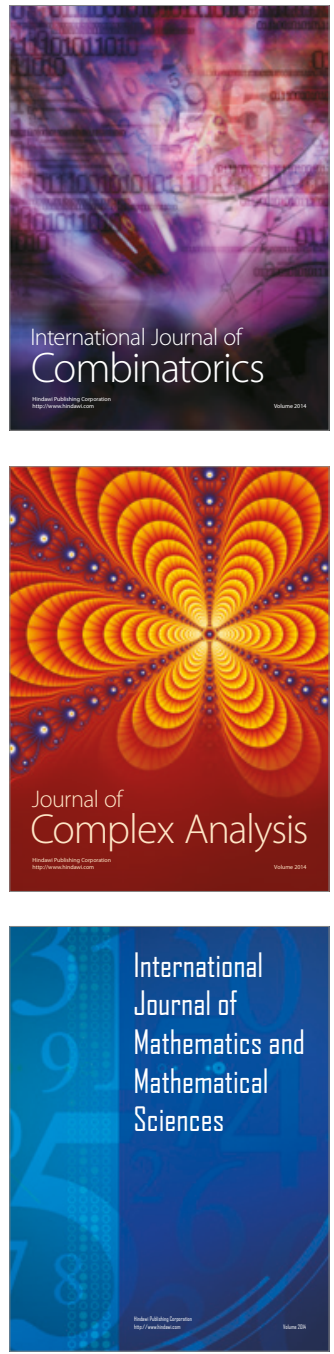
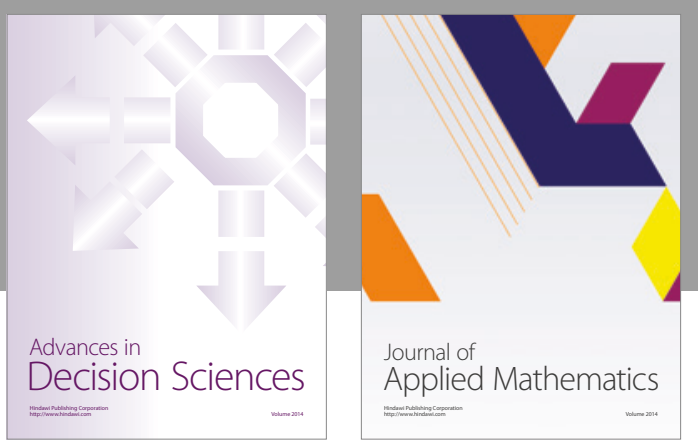

Algebra

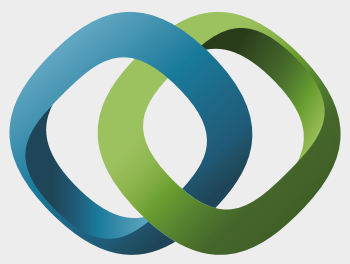

\section{Hindawi}

Submit your manuscripts at

https://www.hindawi.com
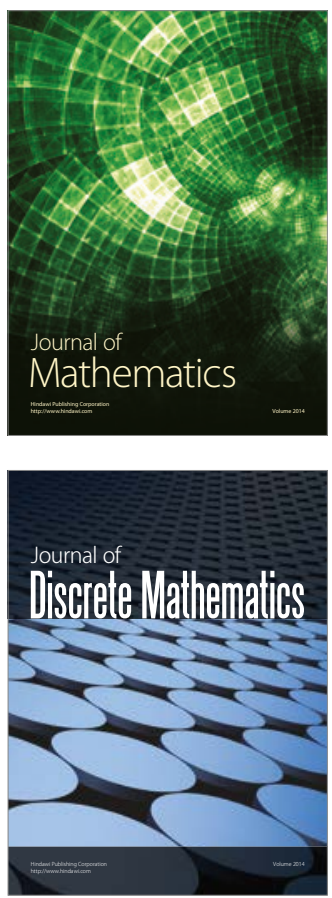

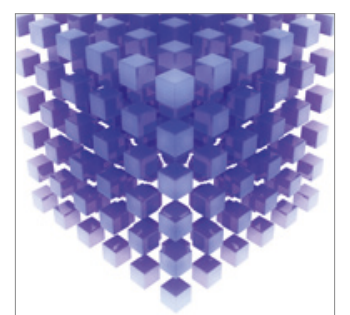

Mathematical Problems in Engineering
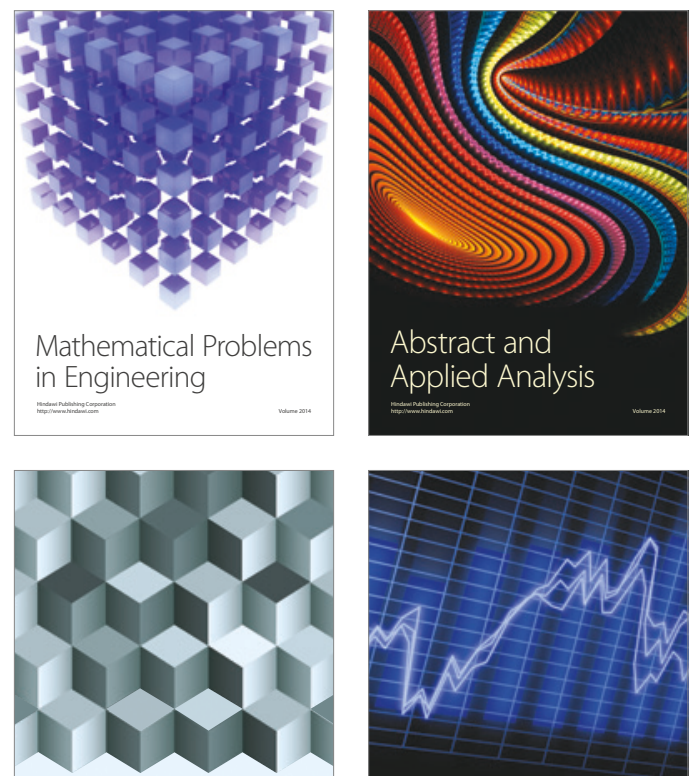

Journal of

Function Spaces

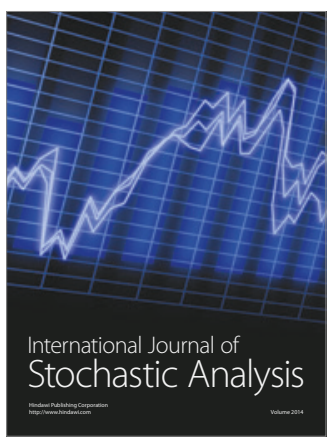

Probability and Statistics
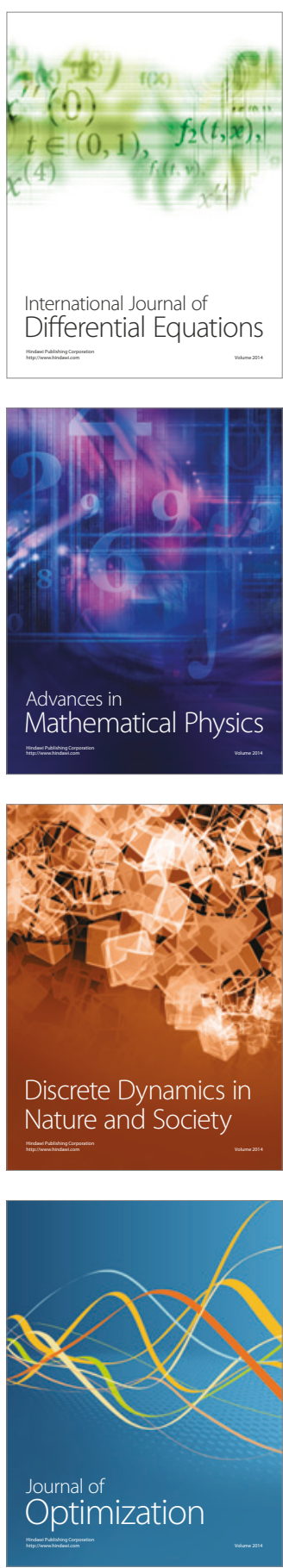